\title{
Parasitisme des petits ruminants dans la zone périurbaine de Sokodé, Togo. II. Caprins
}

\author{
P. Bastiaensen ${ }^{1} *$ P. Dorny ${ }^{2} \mathrm{~K}$. Batawui ${ }^{1}$ \\ A. Boukaya ${ }^{1}$ A. Napala ${ }^{1}$ G. Hendrickx ${ }^{1}$
}

Mots-clés

Caprin - Trypanosoma - Nematoda Hématocrite - Togo.

\section{Résumé}

Une étude parasitologique transversale menée sur 226 échantillons d'origine caprine de la zone périurbaine de Sokodé (région centrale du Togo) a permis le diagnostic de la coccidiose (prévalence de 31 p. 100) et des nématodes gastro-intestinaux (85 p. 100) représentés par Trichostrongylus sp. (29 p. 100), Cooperia sp. (2 p. 100) et Haemonchus sp. (69 p. 100). En outre, des nématodes pulmonaires (Protostrongylus rufescens) ont été rencontrés (36 p. 100), ainsi que le cestode Monieza sp. (3 p. 100). Les trématodes ont été représentés par Paramphistomum sp. (10 p. 100) et Dicrocoelium sp. (un seul échantillon positif). Des trois agents principaux de la gale, seule l'existence de Sarcoptes sp. a pu être confirmée. Les tiques ont été représentées en nombre égal par Amblyomma et Boophilus sp. Des analyses sérologiques ont révélé que 11 p. 100 des animaux étaient séropositifs pour Toxoplasma gondii. La trypanosomose (8 p. 100) demeure la parasitose ayant l'impact le plus important sur les paramètres de santé des caprins, en particulier l'hématocrite. D'autres résultats de cette étude ont été comparés avec ceux des ovins. L'espèce caprine est apparue, d'une manière générale, comme plus rustique et résistante aux pathologies parasitaires rencontrées, notamment la trypanosomose, la toxoplasmose et les nématodoses gastro-intestinales. Plusieurs analyses statistiques qui ont pris en compte des facteurs environnementaux, génétiques et de gestion ont permis d'avoir une meilleure compréhension des interactions hôte-environnement.

\section{INTRODUCTION}

La présente étude a été réalisée dans le cadre du Projet de lutte contre la trypanosomose animale au Togo (Plta). Afin de compléter les travaux extensifs menés sur la filière bovine, cette étude a eu pour but de répertorier les problèmes d'ordre parasitaire auxquels sont confrontés les éleveurs de petits ruminants qui approvisionnent la ville de Sokodé en viande de mouton ou de chèvre (4). Ainsi elle donne suite à l'étude socio-économique de l'élevage de

\footnotetext{
1. Projet régional de lutte contre la trypanosomose animale, Direction nationale, BP 114, Sokodé, Togo.

2. Institut Prince Léopold de médecine tropicale, département de santé et de production animale, Nationalestraat 155, B-2000 Anvers, Belgique

* Auteur pour la correspondance

PO Box 145, 7270 Napier, WC, South Africa

Fax : 27 (28) 4233401 ; e-mail : office@bastiaensen.be
}

petits ruminants dans les zones périurbaines de Lomé et de Sokodé, effectuée dans le cadre du projet Secoville en 1995, sous l'égide de la Conférence des responsables de recherche agronomique africains (Coraf). La deuxième partie de cet article traite de la problématique chez les caprins et des différences avec les ovins.

\section{MATERIEL ET METHODES}

\section{Zone de l'étude}

La ville de Sokodé est, du point de vue démographique, la deuxième ville du Togo avec plus de 100000 habitants. La ville couvre une superficie d'environ $18 \mathrm{~km}^{2}$ avec une densité moyenne de 3372 habitants au $\mathrm{km}^{2}$ (3). La zone périurbaine de Sokodé est délimitée par l'axe de la route nationale 1 (nord-sud) et par l'axe Bassar-Tchamba (ouest-est), ainsi que par la proximité des villes de Kara et d'Atakpamé et enfin par deux réserves de faune, 
la réserve de Fazao-Malfakassa (au sud-ouest de Sokodé) et la réserve de la forêt d'Abdoulaye (au sud-est de Sokodé), dans lesquelles ne se pratique en principe aucune activité économique. Cette zone est ainsi délimitée par un rayon d'environ $50 \mathrm{~km}$ autour de Sokodé et elle regroupe les villages de Blitta, Sotouboua, Lama-Tessi, Tchamba, Kambolé et Bassar.

\section{Elevage}

L'élevage est du type sédentaire, semi-extensif. Les infrastructures présentes sur pratiquement toutes les fermes étaient les parcs de nuit et/ou les abris. Les troupeaux encadrés bénéficiaient d'une amélioration des infrastructures (parcs de contention, râteliers et mangeoires). A peu près la moitié des animaux suivis recevaient une complémentation alimentaire, le plus souvent les épluchures de manioc et les déchets de maïs. Basé sur les réponses des propriétaires, tous les animaux recevaient un soin vétérinaire de contrôle à un moment ou un autre de leur vie, le plus souvent la vaccination contre la peste des petits ruminants (91 p. 100), dans 21 p. 100 des cas un déparasitage interne et externe. La grande majorité des caprins dans les troupeaux visités étaient indigènes, c'est-à-dire de race Naine Ouest-africaine. Les 10 p. 100 d'animaux exotiques (chèvre du Sahel) se trouvaient exclusivement dans des troupeaux encadrés. La répartition des échantillonnages entre les deux sexes reflétait la composition de la plupart des troupeaux (16 p. 100 de mâles contre 84 p. 100 de femelles). Les mâles représentés étaient soit des géniteurs, soit des jeunes animaux au stade de croissance. La pyramide d'âge résumait bien les préférences des éleveurs rencontrés ; les mâles étaient destinés pratiquement exclusivement à l'embouche à partir de l'âge de 14 mois. La sélection des femelles pour l'embouche ou pour la reproduction se faisait apparemment après trois ans.

\section{Animaux}

Les races caprines rencontrées étaient la race Naine de l'Afrique de l'Ouest (type guinéen, trypanotolérant) et la chèvre du Sahel (trypanosensible) $(21,26)$. Selon les résultats du recensement agricole de 1999 (1), il y aurait dans la région centrale 62083 caprins. Basé sur une extrapolation à partir du canevas régulier étant utilisé au Togo pour le Projet de lutte contre la trypanosomose animale au Togo (Plta), le nombre de caprins dans la zone de l'étude avait été évalué à 35800 . L'échantillonnage minimal a été fixé à 300 caprins $(20,22)$, l'objectif ayant été d'obtenir environ la moitié des données dans des troupeaux à gestion améliorée et l'autre moitié dans des troupeaux villageois. Les troupeaux du premier groupe étaient bien connus et répertoriés ; ils bénéficiaient de visites régulières d'un agent vétérinaire ou zootechnicien qui assumait le suivi technique de ces exploitations. Les troupeaux villageois n'étaient pas ou étaient peu connus. Ces derniers ont donc été regroupés suite à une première visite de sensibilisation qui devait amener les propriétaires à participer à l'échantillonnage. Une première série d'échantillonnages s'est déroulée en début de saison sèche (octobre-novembre 1996), une deuxième en début de saison des pluies (avril-mai 1997). Parmi les animaux présentés, seuls les animaux sevrés ont été échantillonnés. Un nombre d'environ 20 têtes par village ou par troupeau a été échantillonné, l'objectif ayant été d'atteindre 150 mâles et 150 femelles d'une part, 150 jeunes et 150 adultes (incisives adultes) d'autre part.

\section{Récolte de données et échantillonnage}

Un examen clinique et une anamnèse ont été effectués sur chaque animal échantillonné dans le but d'évaluer la condition générale de l'animal, de rechercher des symptômes directs ou indirects d'infestation d'ectoparasites et de récolter d'éventuels ectoparasites macroscopiques (poux, tiques) ou de réaliser des raclures dermiques (gale). La prise de sang systémique (environ $8 \mathrm{ml}$ ) a été effectuée à l'aide d'un tube hépariné sous vide (Vacutainer) dans la veine jugulaire. La prise de sang périphérique a été effectuée à l'aide d'un tube capillaire dans une veine auriculaire. Les fèces des moutons ont été récoltées par des techniciens, munis d'un gant en latex ou en plastique. Les matières fécales récoltées dans les gants étaient ensuite transférées dans un bocal en plastique avec couvercle. Chaque échantillon a porté comme identification la localité, la date, l'animal, l'âge, le numéro de l'échantillon.

\section{Techniques d'analyses parasitologiques}

\section{Analyses sérologiques}

La recherche d'anticorps spécifiques contre la toxoplasmose (Toxoplasma gondii) a été réalisée par le laboratoire du Plta à l'aide du kit diagnostic Toxoscreen-DA (agglutination directe).

\section{Hématocrite et protozoologie}

Le principal paramètre clinique retenu a été l'hématocrite pour lequel deux systèmes de prélèvement ont été appliqués, une prise de sang dans un tube capillaire dans la veine auriculaire (sang périphérique) et une prise de sang dans un tube Vacutainer hépariné dans la veine jugulaire. La comparaison des deux techniques a permis de choisir l'hématocrite systémique (jugulaire) comme le plus fiable.

Après centrifugation des microcapillaires héparinés (sang périphérique), le tube a été coupé un millimètre au-dessus de l'interphase caillot blanc/érythrocytes (buffy coat) afin d'y inclure la couche supérieure d'érythrocytes. Le contenu de la fraction supérieure a été étalé sur une lame recouverte d'une lamelle et a été observé au microscope binoculaire (x 40) (18). Un minimum de 50 champs par lame a été examiné. Les frottis sanguins à partir de sang jugulaire ont été fixés et colorés à l'HémaColor ${ }^{\circledR}$ et ensuite observés au microscope binoculaire (10 x 40) (7).

\section{Coprologie}

La coproscopie quantitative a été réalisée à l'aide de la lame de McMaster (microscope 10 x 40), le liquide de flottaison utilisé étant une solution saline dense $(\mathrm{d}=1,20)$ qui ne concentre pas les œufs de trématodes. Ces derniers ont été recherchés à l'aide de la technique de sédimentation (16). Les nématodes gastro-intestinaux ont été différentiés par la technique de la coproculture de larves du stade L3 (23) (microscopie 10 x 40, après traitement au lugol et au lactophenol) (16). Les larves des nématodes pulmonaires ont été récoltées selon la technique de Baermann (13) Les larves ont été identifiées sous microscope suivant les clés fournies par Thienpont et coll.(24).

\section{Parasites externes}

Les tiques ont été arrachées manuellement sur les animaux et identifiées selon les clés établies par Morel (17). Les poux et les puces ont été récoltés manuellement sur les animaux et identifiés, comme pour les raclures dermiques, selon les clés établies par la Maff (16).

\section{Analyse des résultats}

Dans le cadre de cet article, seuls les résultats parasitologiques sont présentés. Ces paramètres ont été analysés par rapport aux facteurs biologiques ou environnementaux recueillis pour les liaisons les plus pertinentes : race, sexe, âge, état physiologique (lactation, gestation, croissance), soins vétérinaires, alimentation, saison. Les analyses statistiques (analyse à un facteur et modèle linéaire généralisé (Glm) ont été réalisées à l'aide du logiciel Spss, version 10.0.5.) (Spss Inc., 1999). 


\section{RESULTATS}

Le nombre de 300 échantillons n'a pas pu être atteint, principalement à cause du nombre réduit de troupeaux encadrés à gestion améliorée. Sur les 226 animaux échantillonnés, 182 provenaient de troupeaux villageois et à peine 44 de troupeaux encadrés par le Plta ou d'autres projets. Etant donné ce déséquilibre, l'aspect encadrement n'a plus été pris en compte comme facteur environnemental. L'influence de ces quelques troupeaux encadrés est apparue toutefois à travers des facteurs comme les soins vétérinaires, la complémentation alimentaire et la race. L'échantillon de 226 animaux était composé de 36 mâles et 190 femelles, 86 jeunes (moins de 14 mois) et 140 adultes.

\section{Protozoaires}

Le tableau I résume les résultats des analyses sanguines en prenant en compte la plus sensible des techniques et l'hématocrite jugulaire. La prévalence moyenne (toutes espèces confondues) a été de 8,41 p. 100. Trois espèces ont été identifiées : Trypanosoma congolense (6,19 p. 100), Trypanosoma vivax (3,54 p. 100) et Trypanosoma melophagium (0,44 p. 100). Sur 19 infections, quatre ont été des infections mixtes $T$. congolense $+T$. vivax.

Une première indication de l'impact de la trypanosomose sur l'hématocrite moyen a été donnée par l'hématocrite moyen des cas positifs qui était de 20,68 p. 100 par rapport à 27,78 p. 100 pour les cas négatifs $(\mathrm{p}<0,001)$. Inversement, la prévalence de la trypanosomose chez les caprins anémiés (ayant eu un hématocrite de moins de 25 p. 100) a été de 17,14 p. 100 au lieu de 8,41 p. 100 en moyenne. Parmi les infections singulières à Trypanosoma vivax et à Trypanosoma congolense, seule l'infection par T. congolense a eu un impact significatif sur l'hématocrite moyen des animaux infectés $(\mathrm{p}<0,001)$ (figure 1). Les infections mixtes n'ont pas eu d'impact significatif sur l'hématocrite moyen. L'impact de la trypanosomose sur l'hématocrite moyen des caprins a été confirmé

\section{Tableau I}

Prévalence de Trypanosoma sp. et hématocrite moyen des cas positifs

\begin{tabular}{lrrr} 
Espèce & N & n & $\%$ \\
\hline T. vivax & 226 & 8 & 3,54 \\
T. congolense & 226 & 14 & 6,19 \\
T. brucei & 226 & 0 & 0,00 \\
T. melophagium & 226 & 1 & 0,44 \\
T. congolense seul & 226 & 10 & 4,42 \\
$\begin{array}{l}\text {. vivax seul } \\
\text { T. congolense + T. vivax }\end{array}$ & 226 & 4 & 1,77 \\
$\begin{array}{l}\text { Trypanosoma sp. } \\
\text { Nb. moyen de trypanosomes }\end{array}$ & 226 & 19 & 1,77 \\
$\begin{array}{l}\text { par champ microscopique } \\
\text { (pour les cas positifs) }\end{array}$ & & & \\
$\begin{array}{l}\text { Prévalence de la trypanosomose } \\
\text { des animaux ayant un hématocrite } \\
\text { jugulaire en dessous de 25 \% }\end{array}$ & 70 & 12 & 17,14 \\
$\begin{array}{l}\text { Hématocrite jugulaire moyen } \\
\text { des cas positifs }\end{array}$ & & \\
\end{tabular}

$\mathrm{N}=$ nombre total d'animaux examinés

$\mathrm{n}=$ nombre d'animaux positifs

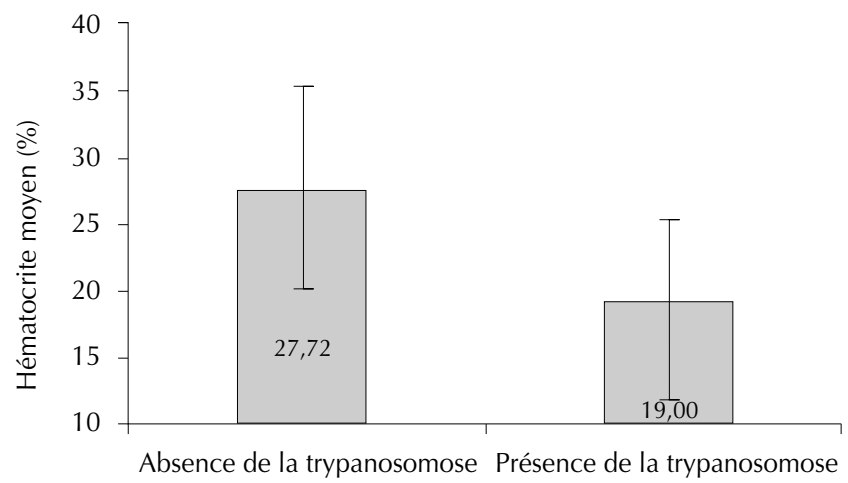

Figure 1 : Trypanosoma congolense.

pour les animaux de race locale Naine ( $\mathrm{p}<0,001$ ), les résultats pour les animaux exotiques ou croisés n'ayant pas été concluants.

Théoriquement, la prévalence de la trypanosomose peut être diminuée par la pratique d'une complémentation alimentaire qui améliore la résistance immunitaire. Ceci avait déjà été noté dans les troupeaux (villageois) ovins mais n'a pas été confirmé chez les caprins pour lesquels aucun effet significatif n'a pu être noté.

Aucune autre hémoparasitose d'ordre protozoaire n'a pu être montrée par les examens des frottis sanguins. Parmi les autres protozooses, on retient la présence de la coccidiose (intestinale), causée par le genre Eimeria. La prévalence a été de 31 p. 100 et la moyenne des taux d'oocystes par gramme de matière fécale (opg) a été de $100,6( \pm 429,9)$.

Des anticorps contre Toxoplasma gondii ont été trouvés chez 11 p. 100 des caprins examinés (titre de 1/40) en saison sèche. Les analyses sérologiques n'ont pas été réalisées en saison des pluies. Contrairement aux ovins, il semblerait que l'âge des animaux ait eu une influence significative sur la prévalence : à l'âge de 2-3 ans, la prévalence a été de 31 p. $100(\mathrm{p}=0,037)$. En outre, sur cinq troupeaux visités, deux étaient indemnes et trois infestés.

\section{Nématodes gastro-intestinaux}

Des microfilaires ont été rencontrées occasionnellement sur les lames à frais ou sur les lames préparées. Leur prévalence apparente a été de 0,88 p. 100 (2 cas sur 226 lames examinées). Les nématodes gastro-intestinaux ont été représentés par Strongyloides sp., la famille des Trichuridae, des Capillariidae, mais surtout des Trichonematidae et des Trichostrongylidae ; parmi ces deux derniers ont été trouvés, par l'examen coprologique et l'identification des larves infectieuses L3, les espèces suivantes : Trichostrongylus sp., Cooperia sp., Oesophagostomum sp. et Haemonchus sp. Les espèces les plus fréquemment rencontrées ont été Haemonchus sp. (69 p. 100) et Trichostrongylus sp. (29 p. 100) (tableau II).

La saison sèche a eu un effet négatif sur les excrétions d'œufs de nématodes et sur la prévalence des infestations gastro-intestinales. Si la prévalence en saison des pluies a été de 98 p. 100, elle a diminué à 72 p. 100 en saison sèche $(p<0,001)$. Les opg moyens ont évolué de 1466 en saison des pluies à 811 en saison sèche $(\mathrm{p}=0,010)$ (tableau III).

\section{Nématodes pulmonaires}

Les nématodes pulmonaires récoltés et identifiés lors des enquêtes en saison sèche appartenaient tous à l'espèce Protostrongylus rufescens. Malgré leur prévalence relativement élevée (36 p. 100), aucun rapport avec des signes cliniques ou l'état de santé des animaux n'a pu être montré (tableau IV). 
Tableau II

Résultats des examens coproscopiques (œufs) et des coprocultures (larves)

\begin{tabular}{lcr} 
Genre & Opg moyen & Ecart-type \\
\hline Strongyloides & 55,2 & 172,90 \\
Trichuris & 8,9 & 49,90 \\
Capillaria & 0,4 & 3,21 \\
\hline Strongles digestifs & $\mathbf{1 1 0 2 , 7}$ & 1637,17 \\
\cline { 2 - 3 } & Espèce (coproculture L3) & \% \\
\cline { 2 - 3 } & Cooperia sp. & 2 \\
& Haemonchus sp. & 69 \\
& Oesophagostomum sp. & 0 \\
& Trichostrongylus sp. & 29
\end{tabular}

Opg $=$ œufs par gramme de matière fécale

Coproculture : détermination réalisée sur 333 larves L3

\section{Tableau III}

Impact de la saison sur le taux d'infestation (opg moyen) et la prévalence (\%) des nématodoses gastro-intestinales

\begin{tabular}{llr} 
Opg moyen & Saison des pluies & 1466 \\
& Saison sèche & 811 \\
Moyenne générale & & 1155 \\
\hline \multirow{2}{*}{ Prévalence } & Saison des pluies & 98 \\
Moyenne générale & Saison sèche & 72 \\
& & 85
\end{tabular}

Opg = œufs par gramme de matière fécale

Saison des pluies $(\mathrm{Glm})$ : opg moyen $(\mathrm{p}=0,010)$; prévalence $(\mathrm{p}<0,0001)$

\section{Cestodes}

Les cestodes ont été représentés exclusivement par le genre Monieza (prévalence de 3 p. 100).

\section{Trématodes}

Les trématodes ont été représentés par deux genres. Le plus fréquemment rencontré a été Paramphistomum sp. (10,45 p. 100). Dicrocoelium sp., la petite douve hépatique, n'a été rencontré qu'une fois (tableau IV). Il s'agit probablement de Dicrocoelium hospes, décrit au Togo par Bourgat et coll. (6).

\section{Ectoparasites}

La seule espèce de gale diagnostiquée a été Sarcoptes sp. (un cas confirmé sur 12 cas douteux).

Les deux genres de tiques rencontrés dans le cadre de cette étude sur les caprins ont été, en nombre égal, Boophilus et Amblyomma. Des larves ont été retrouvées accidentellement dans des digestions de raclures dermiques d'animaux soupçonnés d'avoir des plaies scabieuses. Amblyomma a été récolté essentiellement en saison des pluies alors que Boophilus a été dominant en saison sèche. Malgré un examen minutieux de tous les animaux échantillonnés, presque aucun animal n'a semblé être affecté ni par des poux, ni par des puces. La seule espèce de poux récoltée a été Linognathus africanus (un cas sur 226) et la seule espèce de puces rencontrée a été Ctenocephalides sp. (un cas sur 226) (tableau IV).

\section{Tableau IV}

Tableau récapitulatif de la prévalence (directe ou sérologique) des différentes espèces parasitaires (par catégorie) et comparaison avec les résultats pour les ovins

\begin{tabular}{|c|c|c|c|c|}
\hline & & Capri & & Ovins \\
\hline & $\mathbf{N}$ & $\mathbf{n}$ & $\%$ & $\%$ \\
\hline Protozoaires & & & & \\
\hline Trypanosoma sp. & 226 & 19 & 8 & 13 \\
\hline Eimeria sp. & 179 & 56 & 31 & 33 \\
\hline Toxoplasma gondii & 93 & 10 & 11 & 22 \\
\hline Microfilaires & 2 & 226 & 0,88 & 1,67 \\
\hline $\begin{array}{l}\text { Nématodes } \\
\text { gastro-intestinaux }\end{array}$ & & & & \\
\hline Strongles digestifs & 179 & 153 & 85 & 89 \\
\hline $\begin{array}{l}\text { Nématodes } \\
\text { pulmonaires }\end{array}$ & & & & \\
\hline $\begin{array}{l}\text { Protostrongylus } \\
\text { rufescens }\end{array}$ & 143 & 52 & 36 & 16 \\
\hline Cestodes & & & & \\
\hline Monieza sp. & 179 & 5 & 3 & 8 \\
\hline Trématodes & & & & \\
\hline Fasciola sp. & 67 & 0 & 0,00 & 1,13 \\
\hline Dicrocoelium sp. & 67 & 1 & 1,49 & 0,00 \\
\hline Paramphistomum sp. & 67 & 7 & 10,45 & 15,25 \\
\hline Schistosoma sp. & 67 & 0 & 0,00 & 0,56 \\
\hline Gale & & & & \\
\hline $\begin{array}{l}\text { Indices } \\
\text { macroscopiques }\end{array}$ & 226 & 15 & 6,64 & 3,34 \\
\hline $\begin{array}{l}\text { Confirmation } \\
\text { microscopique }\end{array}$ & 226 & 1 & 0,44 & 1,39 \\
\hline Sarcoptes & 12 & 1 & 8 & 15 \\
\hline Chorioptes & 12 & 0 & 0 & 15 \\
\hline Psoroptes & 12 & 0 & 0 & 8 \\
\hline Tiques & & & & \\
\hline Prélèvements & 226 & 14 & 6 & 11 \\
\hline Amblyomma & 14 & 7 & 50 & 13 \\
\hline Rhipicephalus & 14 & 0 & 0 & 0 \\
\hline Boophilus & 14 & 7 & 50 & 87 \\
\hline Poux & & & & \\
\hline $\begin{array}{l}\text { Linognathus } \\
\text { africanus }\end{array}$ & 226 & 1 & 0,44 & 0,28 \\
\hline Puces & & & & \\
\hline Ctenocephalides sp. & 226 & 1 & 0,44 & 0,00 \\
\hline
\end{tabular}

$\mathrm{N}=$ nombre total d'animaux examinés

$\mathrm{n}=$ nombre d'animaux positifs

\section{DISCUSSION ET COMPARAISON} DES RESULTATS AVEC L'ESPECE OVINE

De façon générale l'encadrement sanitaire et zootechnique des ovins a été nettement supérieur à celui des caprins : au niveau de l'encadrement par des structures comme des projets ou des programmes, des infrastructures, de l'habitat et du suivi vétérinaire, les caprins ont été moins bien représentés que les ovins.

Les agents parasitaires identifiés dans ces deux études ont été trouvés de façon générale aussi bien chez les ovins que chez les 
caprins, sauf dans quelques cas de prévalences extrêmement faibles. La prévalence moyenne de la trypanosomose chez les ovins et les caprins a été similaire : 13,1 p. 100 chez les ovins et 8,4 p. $100 \mathrm{chez}$ les caprins (différence non significative ; $\mathrm{p}=0,0814)$. La prévalence moyenne chez les bovins dans la même zone périurbaine était de 9,43 p. 100 (15). Ces résultats sont légèrement supérieurs aux résultats des enquêtes protozoologiques que le Plta avait mené dans le cadre de ses opérations de lutte dans les années 1990-1993 (14) et qui présentaient une prévalence de 8,72 p. 100 pour les ovins et de 5,99 p. 100 pour les caprins. Trois espèces ont été identifiées : Trypanosoma congolense, T. vivax et, à une seule occasion, T. melophagium. La prévalence de la trypanosomose animale chez les caprins de la race Naine Ouestafricaine au Nigeria (10) était de 13,6 p. 100, avec une majorité de T. brucei (50 p. 100).

Des anticorps contre Toxoplasma gondii ont été trouvés chez 22 p. 100 des ovins et 11 p. 100 des caprins examinés en saison sèche. La présence de la toxoplasmose a semblé être surtout liée aux troupeaux, plutôt qu'aux animaux individuels. Ainsi, il a été trouvé dans certains troupeaux une prévalence de 68 p. 100.

Les résultats sur les nématodes gastro-intestinaux confirment les observations de Fakae (11) pour le Nigeria et de Bonfoh et coll. (5) pour la région des plateaux au Togo (à travers de nécropsies), à l'exception d'Oesophagostomum sp. et de Gaigeria sp. qui n'ont pas été observés dans la présente étude chez les caprins, ce qui n'empêche que ces nématodes peuvent être présents à de faibles densités (moins de $50 \mathrm{opg}$ ), avec toujours un impact pathogène (12). Les résultats ont aussi confirmé les différences en sensibilité entre les deux espèces : les opg moyens ont été nettement plus élevés chez les ovins (différence significative ; $p=0,0002$ ). En revanche, la prévalence a été pratiquement la même (85 p. 100 chez les caprins, 89 p. 100 chez les ovins, $\mathrm{p}=0,33$ ). Pour la région des plateaux, Bonfoh et coll. (5) n'ont pas pu montrer de différences significatives entre la charge parasitaire des ovins et des caprins. En revanche, ils font état de l'influence prépondérante de la saison sur les opg. Dans les deux études présentes, l'influence de la saison a été très significative pour les caprins, aussi bien au niveau des opg qu'au niveau de la prévalence ; elle ne l'a pas été chez les ovins où elle a influencé seulement la prévalence de façon significative. En revanche, l'effet combiné des facteurs sexe, âge et état physiologique observé chez les ovins, et probablement lié au phénomène d'augmentation des opg lors du péripartum, a été absent ou du moins non significatif chez les caprins, ce qui a d'ailleurs été constaté par plusieurs auteurs $(9,25)$. Seuls Baker et coll. (2) font état d'une hausse significative de l'opg durant la lactation chez des chèvres de race Galla et Small East African.

L'impact de l'infestation par les nématodes gastro-intestinaux sur l'hématocrite des animaux n'a pas été significatif, bien que la tendance ait été visible. Chez les caprins, aucune combinaison n'a permis de montrer un impact significatif de l'opg sur l'hématocrite; chez les ovins, ce n'est que lorsque les différents taux étaient classés selon leur impact pathologique et spoliateur sur l'animal (niveau faible, moyen ou élevé) que cette relation était significative. Une corrélation linéaire et significative n'a été observée que pour les ovins à partir de l'âge de trois ans. Les mêmes difficultés ont été rencontrées ailleurs par d'autres auteurs. Selon Costa et coll. (8), l'opg (après transformation logarithmique) serait mieux corrélé avec le taux d'hémoglobine qu'avec l'hématocrite.

L'effet synergique observé pour les infections par les trypanosomes, d'une part, et les nématodes gastro-intestinaux, d'autre part, sur l'hématocrite moyen a pu être clairement montré chez les ovins ; il y a peu de doutes que le même effet se produit chez les caprins. Malheureusement, le nombre réduit de données pour cer- taines classes chez les caprins a empêché l'élaboration d'un graphique similaire pour cette catégorie d'animaux.

En ce qui concerne la gale, finalement, le seul résultat positif (microscopie) a confirmé les observations macroscopiques (cliniques) qui semblaient indiquer une prépondérance de Sarcoptes scabiei var. capri chez la chèvre, comme observé ailleurs en Afrique occidentale (19), alors que les trois espèces (Sarcoptes, Chorioptes et Psoroptes sp.) ont été trouvées chez les ovins.

\section{CONCLUSION}

Les résultats de cette étude chez les caprins ont confirmé en grande partie les tendances observées chez les ovins de la même zone : la trypanosomose animale reste le fléau principal des petits ruminants, avant tout des ovins qui semblent être plus sensibles aux maladies parasitaires (en termes de prévalence, de niveau d'infestation et d'effets sur la santé) que les caprins (indigènes). En effet, aussi bien la prévalence de la trypanosomose que l'intensité de l'infestation (opg) des nématodoses gastro-intestinales ont été sensiblement plus élevées chez les ovins que chez les caprins, ce qui se reflète aussi dans l'hématocrite moyen qui est significativement plus bas chez les ovins que chez les caprins (respectivement 24,10 et 27,$10 ; \mathrm{p}<0,0001)$.

Dans l'optique de l'approvisionnement de la ville de Sokodé par ces exploitations périurbaines, les conclusions de cette étude doivent évidemment être complétées par d'autres études vétérinaires (la situation de la peste des petits ruminants, par exemple) et une étude zootechnique, permettant de mettre en rapport les contraintes sanitaires avec les performances en matière de production et de reproduction. Seule une appréciation large de l'élevage périurbain pourra contribuer à une meilleure compréhension et, par conséquence, à une intervention dans le secteur.

\section{Remerciements}

Les auteurs remercient chaleureusement tous les éleveurs, collaborateurs et techniciens qui ont participé à cette étude. Les auteurs souhaitent aussi remercier les docteurs K. Adoméfa, B. Faye, K. Tessio, Y. Pessinaba, A. Traoré, J. Slingenbergh et A. Sonhaye, MM. J.D. Keita, A. Rakotomahefason, K. Amedji et T. Lemou, et les professeurs V.S. Pandey et A. Verhulst. Enfin, il convient de remercier l'ONU-FAO, qui exécuta le projet Plta, et la Belgique, principal bailleur de fonds dudit projet.

\section{BIBLIOGRAPHIE}

1. Aperçu de I'agriculture togolaise à travers le pré-recensement, 1997. Projet GCP/TOG/014-EC recensement national agricole. Lomé, Togo, direction des Enquêtes et statistiques agricoles.

2. BAKER R.L., MWAMACHI D.M., AUDHO J.O., ADUDA E.O., THORPE W., 1998. Resistance of Galla and small East African goats in the sub-humid tropics to gastrointestinal nematode infections and the peri-parturient rise in faecal egg counts. Vet. Parasitol., 79: 53-64.

3. BARBIER J-C., KLEIN B., 1995. Petit atlas urbain : Sokodé, ville multicentrée du Nord-Togo. Paris, France, Orstom.

4. BASTIAENSEN P., DORNY P., BATAWUI K., BOUKAYA A., NAPALA A., HENDRICKX G., 2003. Parasitismes des petits ruminants en zone périurbaine de Sokodé, Togo. I. Ovins. Revue Elev. Méd. vét. Pays trop., $56: 43-50$.

5. BONFOH B., ZINSSTAG J., ANKERS P., PANGUI L.J., PFISTER K., 1995. Epidémiologie des nématodes gastro-intestinaux chez les petits ruminants dans la région des plateaux au Togo. Revue Elev. Méd. vét. Pays trop., 48 : 321-326.

6. BOURGAT R., SEGUIN D., BAYSSADE-DUFOUR C., 1975. New data on Dicrocoelium hospes Looss, 1907: anatomy of the adult and life cycle. Preliminary paper. Ann. Parasitol. Hum. comp., 50: 701-714. 
7. BOYT W.P., 1986. Guide pratique pour le diagnostic, le traitement et la prévention de la trypanosomiase animale africaine. Rome, Italie, FAO. 8. COSTA C.A., VIEIRA L.D., BERNE M.D., SILVA M.U., GUIDONI A.L., FIGUEIREDO E.A., 2000. Variability of resistance in goats infected with Haemonchus contortus in Brazil. Vet. Parasitol., 88: 153-158.

9. DORNY P., SYMOENS C., VERCRUYSSE J., JALILA A., SANI R., 1993. Epidemiology of strongyles of small ruminants grazing in a rubber plantation in Malaysia. In: Sivaraj S., Agamuthu P., Mukherjee T.K. Eds, Proc. Workshop on Development of Sustainable Integrated Smal Ruminant - Tree Cropping Production Systems, Nov. 3 - December 4, 1992. Kuala Lumpur, Malaya, IDRC.

10.FAKAE B.B., CHIEJINA S.N., 1993. The prevalence of concurrent trypanosome and gastrointestinal nematode infections in West African

Dwarf sheep and goats in Nsukka area of eastern Nigeria. Vet. Parasitol., 49: 313-318.

11.FAKAE B.B., 1990. The epidemiology of helminthosis in smal ruminants under the traditional husbandry system in eastern Nigeria. Vet. Res. Commun., 14: 381-391.

12.GRABER M., PERROTIN G., 1983. Helminthes et helminthoses de ruminants domestiques d'Afrique tropicale. Paris, France, Editions du Point vétérinaire, $162 \mathrm{p}$.

13. HANSEN J., PERRY B., 1995. Epidémiologie, diagnostic et prophylaxie des helminthiases des ruminants domestiques. Rome, Italie, FAO, 176 p.

14.HENDRICKX G., NAPALA A., 1999. Le contrôle de la trypanosomose à la carte : une approche intégrée basée sur un système d'information géographique. Bruxelles, Belgique, Académie royale des Sciences d'Outre-Mer, p. 88. (Mémoires classe sciences naturelles, médicales, Sér. 8, vol. 24)

15.HENDRICKX G., 1999. Georeferenced decision support methodology towards trypanosomosis management in West Africa. Thèse Doct. faculté de Médecine vétérinaire, université de Gand, Belgique, 176 p.

\section{Summary}

Bastiaensen P., Dorny P., Batawui K., Boukaya A., Napala A., Hendrickx G. Small Ruminant Parasitism in the Suburban Area of Sokode, Togo. II. Goats

The cross-section parasitological analysis of 226 samples taken from goats in the suburban area of Sokode (central area of Togo) enabled the diagnosis of coccidiosis $(31 \%$ prevalence) and gastrointestinal nematodes (85\%) such as Trichostrongylus sp. (29\%), Cooperia sp. (2\%) and Haemonchus sp. (69\%). Furthermore, Protostrongylus rufescens lungworms were encountered $(36 \%)$ as well as the cestode Monieza sp. (3\%). Trematods were represented by Paramphistomum sp. (10\%) and Dicrocoelium sp. (only one positive sample). Of the three major scabies parasites, only Sarcoptes sp. was encountered. Ticks were equally represented by Amblyomma and Boophilus sp. Serological analyses revealed the presence of $11 \%$ positive animals for Toxoplasma gondii. Trypanosomosis ( $8 \%$ ) was still the main parasitological constraint in terms of impact on health parameters, more particularly on PCV. Other results of this study were compared to those obtained in sheep. Goats, as a species, would appear to be more rustic and resistant against parasite-borne pathologies, particularly trypanosomosis, toxoplasmosis and gastrointestinal nematode infestations. Several statistical analyses that took into account environmental, management and breed-related factors allowed better understanding of host-environment interactions.

Key words: Goat - Trypanosoma - Nematoda - Hematocrit Togo.
16. Manual of veterinary parasitological laboratory techniques, 1986. London, UK, Ministry of Agriculture, Fisheries and Food / HMSO, p. 37-39.

17. MOREL P.C., 1976. Morphologie, biologie et rôle pathogène des tiques. Maisons-Alfort, France, Gerdat-lemvt, p. 6-13.

18. MURRAY M., MURRAY P.K., MCINTYRE W.I.M., 1977. An improved parasitological technique for the diagnosis of African trypanosomiasis. Trans. R. Soc. trop. Med. Hyg., 71: 325-326.

19. OLUBUNMI P.A., 1995. The prevalence of caprine sarcoptic mange due to Sarcoptes scabiei var. capri in Ife-Ife area of Nigeria, its control and management. Bull. Anim. Health Prod. Afr., 43: 115-119.

20.PARKER R.E., 1991. Introductory statistics for biology. Cambridge, UK, Cambridge Úniversity Press.

21.PAYNE W.J.A., 1990. An introduction to animal husbandry in the tropics. London, UK, Longman Scientific and Technical, p. 499-500.

22.PUTT S.N.H., SHAW A.P.M., WOODS A.J., TYLER L., JAMES A.J., 1987. Veterinary epidemiology and economics in Africa; a manual for use in the design and appraisal of livestock health policy. ILCA Manual 3. Addis Ababa, Ethiopia, ILCA, 130 p.

23.SKERMAN K.D., HILLARD J.J., 1966. A handbook for studies of helminth parasites of ruminants. Rome, Italy, UNDP/FAO, p. C.VII.1-4.

24.THIENPONT D., ROCHETTE F.R., VANPARIJS O.F.J., 1979. Diagnosis of verminosis by coprological examinations. Beerse, Belgium, Janssen Research Foundation, p. 48-67.

25.VAN AKEN D., DE BONT J., VERCRUYSSE J., DORNY P., 1990. Gastro-intestinal nematode infections in a goat-breeding farm in northwestern Sri Lanka. Trop. Anim. Health Prod., 22: 231-238.

26.WILSON T., 1992. Petits ruminants : production et ressources génétiques en Afrique tropicale. Rome, Italie, FAO, p. 101-139.

Reçu le 15.07.2002, accepté le 23.03.2004

\section{Resumen}

Bastiaensen P., Dorny P., Batawui K., Boukaya A., Napala A., Hendrickx G. Parasitismo de los pequeños rumiantes en la zona peri urbana de Sokodé, Togo. II. Caprinos

Se llevó a cabo un estudio parasitológico transversal, sobre 226 muestras de origen caprino de la zona peri urbana de Sokodé (región central de Togo) permitiendo el diagnóstico de la coccidiosis (prevalencia de $31 \%$ ) y de los nemátodos gastrointestinales (85\%) representados por Trichostrongylus sp. (29\%), Cooperia sp. (2\%) y Haemonchus sp. (69\%). Por otro lado, se encontraron nemátodos pulmonares (Protostrongylus rufescens, $36 \%$ ), así como Monieza sp. (3\%). Los tremátodos fueron representados por Paramphistomum sp. (10\%) y Dicrocoelium sp. (una sola muestra positiva). De los tres agentes principales de la sarna, solo la existencia de Sarcoptes sp. pudo ser confirmada. Las garrapatas fueron representadas en números iguales por Amblyomma y Boophilus sp. Los análisis serológicos revelaron que $11 \%$ de los animales eran seropositivos para Toxoplasma gondii. La tripanosomosis (8\%) se mantiene como la parasitosis con el impacto más importante sobre los parámetros de salud de los caprinos, en particular del hematocrito. Otros resultados de este estudio fueron comparados con los de los ovinos. La especie caprina apareció, de forma general, más rústica y resistente a las patologías parasitarias encontradas, sobre todo la tripanosomosis, la toxoplasmosis y los nemátodos gastrointestinales. Diversos análisis estadísticos, que tomaron en cuenta los factores ambientales, genéticos y de manejo, permitieron una mejor comprensión de las interacciones huésped - medio ambiente.

Palabras clave: Caprino - Trypanosoma - Nematoda Hematocrito - Togo. 